\title{
Substrate Temperature Effects on DC Sputtered Mo thin film
}

\author{
Heejin Ahn, Dongchan Lee, and Youngho Um* \\ Department of Physics, University of Ulsan, Ulsan 44610, Korea
}

Received December 21, 2016; revised January 16, 2017; accepted January 18, 2017

\begin{abstract}
To improve the adhesion of Mo thin film as a back contact material, a DC magnetron sputtering system was used to deposit in the form of a bi-layer on soda-lime glass. Films with low resistivity and good adhesion were obtained from this deposition, even though the two qualities were found be hard to obtain at the same time. The best Mo bilayer showed a resistivity of $8.13 \times 10^{-4} \Omega \cdot \mathrm{cm}$ at $500^{\circ} \mathrm{C}$ and $3.0 \times 10^{-3}$ Torr. The XRD measurements showed that the crystallites of the films were mainly oriented in the (110) direction, the FE-SEM images revealed that the resistivity of the Mo films decreased with increasing substrate temperature, which temperature reduction is accompanied by an increase of the grain size. These experimental results were analyzed using the Fuchs-Sondheimer theory. Our Mo bilayer film with better crystallinity and lower resistivity can be suitably used as a back-contact layer for CIGS solar cells.
\end{abstract}

Keywords: Mo magnetron sputtering, Bi-layer, Electrical Properties, Back-contact layer, CIGS solar cells

\section{Introduction}

Thin film photovoltaics (PV) is an emerging worldwide industry. Due to the recent increase in energy prices, and with growing global ecological concerns, PV has increasingly become a more important technology. From all the current PV technologies, the one that is demonstrating the most industrial interest is $\mathrm{Cu}(\mathrm{In}, \mathrm{Ga}) \mathrm{Se}_{2}$ (CIGS) based photovoltaics. CIGS is one of the most attractive candidates for thin film photovoltaic devices because it is a direct-gap semiconductor with an appropriate band gap of around $1 \mathrm{eV}$; it has one of the highest known absorption coefficients for solar radiation for any semiconductor [1]. CIGS thin film solar cells have several layers; each layer has a different role in working cells. The back-contact in a photovoltaic device generally comprises a metal layer on the bottom of the absorber material; the role of this layer is to collect carriers from the absorber and deliver them to the external load. Basically, a good back-contact material is a metal that can play the above role without carrier loss or detrimental influence on device performance. There are a few other requirements for this layer, such as having low resistivity, good adhesion to SLG, low roughness, chemically inertness with regard to the materials deposited on top, a temperature expansion coefficient similar to those of SLG and CIGS and the ability to form an Ohmic contact with CIGS.

One material that suits almost all the above requirements is molybdenum (Mo), it provides a unique back-contact material for CIGS [2] and has microstructures that can be

*Corresponding author

E-mail:yhum@ulsan.ac.kr from dense to porous depending on the sputter processing parameters such as the deposition pressure, substrate bias and temperature; this variability of the microstructure results in variations in the residual stress, electrical resistivity and film adhesion [3-5]. Sputtering working pressure influences the properties of sputtered atoms in two main ways [6,7]. The first is by changing the deposition rate and the second is the mean free path. With more pressure in the chamber, the probability of a sputtered atom colliding with an Ar atom increases, thus changing the energy of the sputtered atoms. The energy with which the sputtered atoms arrive at the substrate is an important factor that determines the stress state of the films. When depositing Mo thin films under these processing conditions, the resistivity and the adhesion of the films have opposite effects, making it difficult to have a film with both properties maximized. The reason why this happens is usually attributed to the film stress state $[8,9]$. Films that are deposited under compressive stress have low resistivity; they also have low adhesion. On the other hand, films that are deposited under tensile stress have good adhesion but high resistivity.

In this study, to obtain low resistive and good adhesive Mo layers, we have grown Mo bi-layer films in two steps and investigated the effects of substrate temperature on the structural, electrical and morphological properties of the films, where were deposited on soda-lime glass using a DC magnetron sputtering system.

\section{Experiment}

Mo thin films were grown on soda-lime glass using DC 
Table 1. Deposition parameters of the DC magnetron sputtering for preparation of the Mo bi-layer films at $50 \mathrm{~W}$ of the power density (B: bottom layer, $T$ : top layer).

\begin{tabular}{cccc}
\hline \hline $\begin{array}{c}\text { Substrate temp. } \\
\left({ }^{\circ} \mathrm{C}\right)\end{array}$ & $\begin{array}{c}\text { Deposition } \\
\text { Time }(\mathrm{s})\end{array}$ & $\begin{array}{c}\text { Thickness } \\
(\mathrm{m})\end{array}$ & $\begin{array}{c}\text { Deposition } \\
\text { Rate }(\mathrm{nm} / \mathrm{s})\end{array}$ \\
\hline \multirow{2}{*}{200} & $\mathrm{~B}: 6720$ & $300 \mathrm{~nm}$ & \\
& $\mathrm{~T}: 7200$ & $1.06 \mu \mathrm{m}$ & 0.1055 \\
300 & $\mathrm{~B}: 6720$ & $341 \mathrm{~nm}$ & \\
& $\mathrm{~T}: 7200$ & $1.13 \mu \mathrm{m}$ & 0.1095 \\
400 & $\mathrm{~B}: 6720$ & $373 \mathrm{~nm}$ & \\
& $\mathrm{~T}: 7200$ & $1.21 \mu \mathrm{m}$ & 0.1162 \\
500 & $\mathrm{~B}: 6720$ & $422 \mathrm{~nm}$ & \\
& $\mathrm{~T}: 7200$ & $1.39 \mu \mathrm{m}$ & 0.1344 \\
\hline
\end{tabular}

magnetron sputtering at different substrate temperatures with an Mo target (99.9\% purity, 2" in diameter). All SLG had been degreased in organic solution, rinsed in deionized water and blown dry with $\mathrm{N}_{2}$ gas before being loaded into a vacuum chamber. High-purity Ar gas was introduced through a mass flow controller after the vacuum chamber had been evacuated below $3.0 \times 10^{-6}$ Torr. A DC sputter gun with Mo target was mounted downward of the sputter chamber top plate. The distance between the target and the substrate was kept at $11 \mathrm{~cm}$. To grow a good adhesive bottom layer, we deposited Mo film under a working pressure in the chamber of $1.4 \times 10^{-2}$ Torr and consecutively deposited low resistive top layer under $3.0 \times 10^{-3}$ Torr. We kept the substrate spinning during the sputtering process in order to obtain a uniform film. Depositions were performed with $50 \mathrm{~W}$ of DC power. Table I shows the deposition parameters of the DC magnetron sputtering for preparation of the Mo bi-layer films.

The structural properties of the films were examined using X-ray diffraction (XRD). The surface morphology and the thickness of the films were examined using field emission scanning electron microscopy (FE-SEM). Resistivity and carrier concentration were measured in the van der Pauw configuration using a Hall effect measurement system (HMS 5000, ECOPIA21). Finally, we simply checked the adhesion of the Mo bi-layer films to the substrate by detaching Scotch tape.

\section{Results and Discussion}

Figure 1 shows the X-ray diffraction (XRD) spectra indicating that all of the Mo thin films have a typical cubic structure, showing a peak at $40.57^{\circ}$ and a small peak at $73.37^{\circ}$, which correspond to the (110) plane and the (211) plane reflections. The two peaks for the Mo bi-layer are stronger than those for the Mo single layer. The higher the substrate temperatures are, the sharper and stronger the diffraction peaks of the Mo films are, indicating the improved crystallinity. This is likely due to the increase of the film thickness. In addition, the full-width at halfmaximum (FWHM) of the Mo (110) peak decreased with (a)

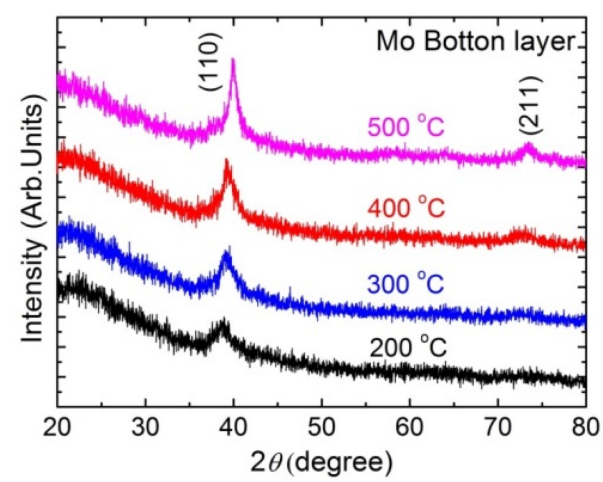

(b)

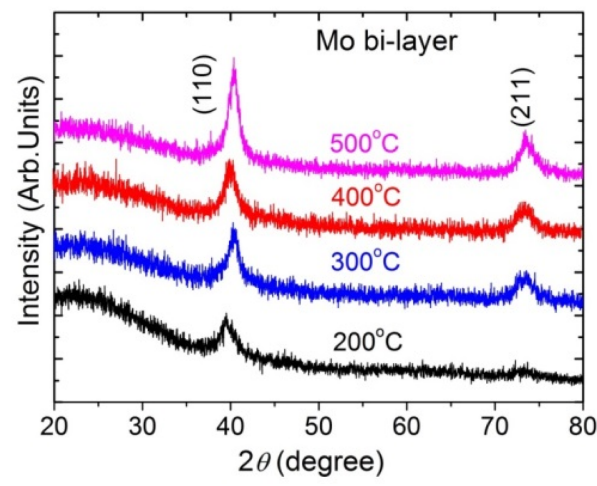

Figure 1. X- ray diffraction spectra of the Mo thin films (a) single layer (b) bi-layer. XRD spectra indicate that all of the Mo thin films have a typical cubic structure, showing a peak at $40.46^{\circ}$ and a small peak at $73.75^{\circ}$ which correspond to the (110) plane and the (211) plane reflections, respectively.

increasing substrate temperature. The lattice parameter $(a)$ is determined for the cubic structure using the following expression:

$$
\frac{1}{d^{2}}=\frac{\left(h^{2}+k^{2}+l^{2}\right)}{a^{2}}
$$

where $h, k$ and $l$ are the Miller indices of the lattice planes. Fig. 2 shows the lattice constants of the Mo thin films as a function of the substrate temperature. The change in lattice constant for the thin film deposited on the substrate clearly suggests that the thin film's grains are under stress; this may be due to a change in the nature and concentration of the native imperfections. This causes either tensile strain or compression strain of the lattice parameters. The density of the film is, therefore, expected to change in accordance with changes in the lattice constants. The lattice constant of the Mo thin film increases with increasing growth temperature. The linear thermal expansion coefficient is related to the effects of the anharmonic terms in the potential energy on the mean separation of a pair of atoms.

The strain on the film was evaluated using the following 


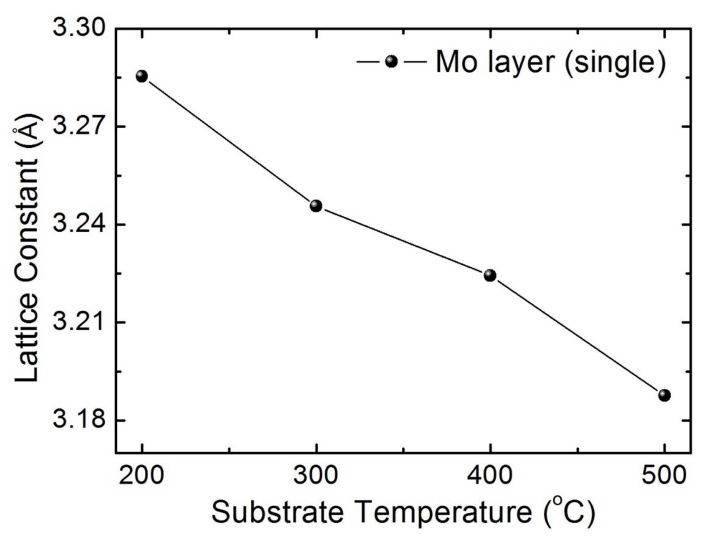

Figure 2. Lattice constants of the Mo thin films as a function of substrate temperature.

formula:

$$
\operatorname{strain}(\%)=\frac{\Delta a}{a} \times 100
$$

where $a$ is the lattice parameter (for Mo, $a=$ $0.31472 \mathrm{~nm}$ ). Lattice constants, grain size, and resistivity of the Mo layer at various substrate temperatures are listed in Table II. Fig. 3 shows the substrate temperature dependence of the strain in the Mo bi-layer.

At low substrate temperatures, Mo films have a more porous columnar grain growth causing intergranular voids. This happens due to the reduced energy of the sputtered atoms that arrive at the substrate. In terms of film properties, these voids make the resistivity higher, but there is no reorganization of the arriving atoms. On the other hand, at high substrate temperature, Mo films have the tendency to become tightly packed; this tends to form films with a compressive stress and there is a decrease in resistivity. The residual strain is changed from tensile to compressive, as the substrate temperature increases. Films deposited at lower temperatures tend to be more resistive, but these films demonstrate good adhesion between the Mo film and the substrate, because the films are subject to a tensile strain, while films deposited at higher substrate temperatures tend to be subject to a compressive strain and demonstrate low resistivity and poor adhesion to the glass substrate [10].

FE-SEM images of the Mo bi-layers deposited under various substrate temperatures are illustrated in Fig. 4. Film prepared at $200^{\circ} \mathrm{C}$ with deposition pressure of $1.4 \times 10^{-2}$ and $3.0 \times 10^{-3}$ Torr showed a smooth surface with fibrous grains of small size, and small size pores, as shown in Fig. 4(a). The inset of the plane-view shows an FE-SEM image of the first Mo layer.

As the deposition temperature of the substrate increased, the pore size became larger and the fibrous grain size increased. This increase in grain size is likely to be the reason for the reduction in the resistivity of the Mo thin films with increasing of the deposition substrate temperature, as can be seen in Fig. 5.
Table 2. Parameters and results of Mo bi-layer deposition at $1.4 \times 10^{-2} / 3.0 \times 10^{-3}$ Torr. (B: bottom layer, T: top layer).

\begin{tabular}{|c|c|c|c|c|}
\hline $\begin{array}{c}\text { Substrate } \\
\text { Temperature } \\
\left({ }^{\circ} \mathrm{C}\right)\end{array}$ & $\begin{array}{c}\text { Lattice } \\
\text { Constant }(\AA)\end{array}$ & $\begin{array}{c}\text { Strain } \\
(\%)\end{array}$ & $\begin{array}{l}\text { Grain Size } \\
\quad(\mathrm{nm})\end{array}$ & $\begin{array}{l}\text { Resistivity } \\
(\Omega \cdot \mathrm{cm})\end{array}$ \\
\hline 200 & 3.1423 & 4.553 & 68.32 & $\begin{array}{l}\text { B: } 1.95 \times 10^{-2} \\
\text { T: } 4.49 \times 10^{-3}\end{array}$ \\
\hline 300 & 3.1494 & 3.054 & 75.12 & $\begin{array}{l}\text { B: } 1.07 \times 10^{-2} \\
\text { T: } 4.08 \times 10^{-3}\end{array}$ \\
\hline 400 & 3.1635 & 1.925 & 86.83 & $\begin{array}{l}\text { B: } 4.75 \times 10^{-3} \\
\text { T: } 2.69 \times 10^{-3}\end{array}$ \\
\hline 500 & 3.2258 & 1.202 & 92.35 & $\begin{array}{l}\text { B: } 1.64 \times 10^{-3} \\
\text { T: } 8.13 \times 10^{-4}\end{array}$ \\
\hline
\end{tabular}

(a)

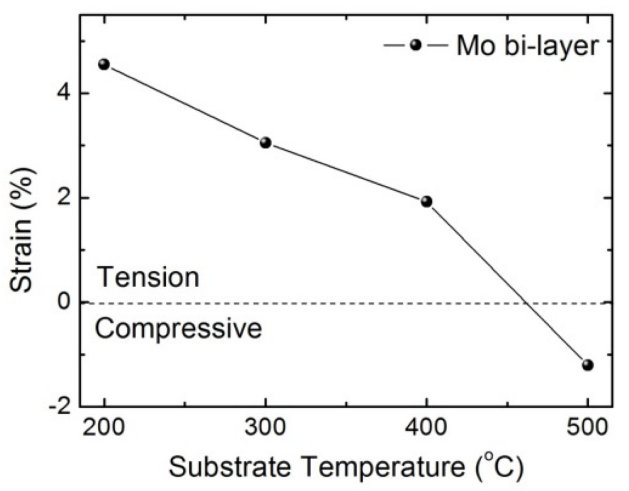

(b)

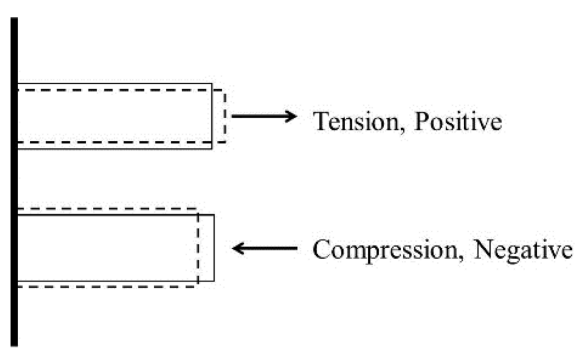

Figure 3. (a) Substrate temperature dependence of the strain in the Mo bi-layer. (b) Direction and sign of the applied force by convention.

Table 2 shows the resistivity of single layer and bi-layer as a function of substrate temperature. Resistivity of all layers tended to decrease with increasing growth temperature. Bilayer shows that the resistivity is lower than single layer.

Electrical resistivity, carrier mobility and carrier concentration dependencies for the Mo bi-layers at various substrate temperatures, measured by Hall measurements at room temperature, are shown in Fig. 5. resistivity decreased from $4.49 \times 10^{-3}$ to $8.13 \times 10^{-4} \Omega \cdot \mathrm{cm}$. The lowest resistivity was $8.13 \times 10^{-4} \Omega \cdot \mathrm{cm}$ for the film with the $500^{\circ} \mathrm{C}$ substrate temperature. Corresponding mobility increased from 0.023 to $1.05 \mathrm{~cm}^{2} / \mathrm{V} \cdot \mathrm{s}$, and carrier density slightly increased from $6.17 \times 10^{21}$ to $6.13 \times 10^{22} \mathrm{~cm}^{-3}$. The electrical resistivity of thin films, according to the Fuch-Sondheimer (FS) theory, 


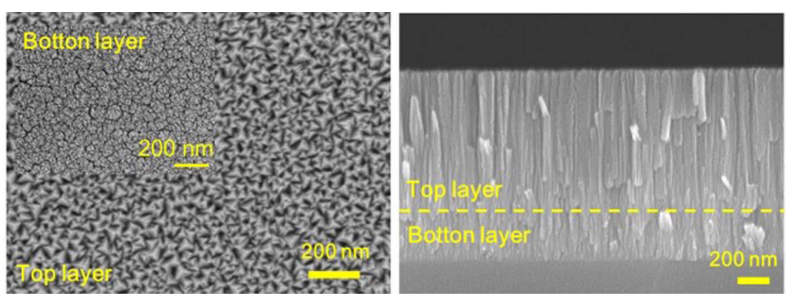

(a)

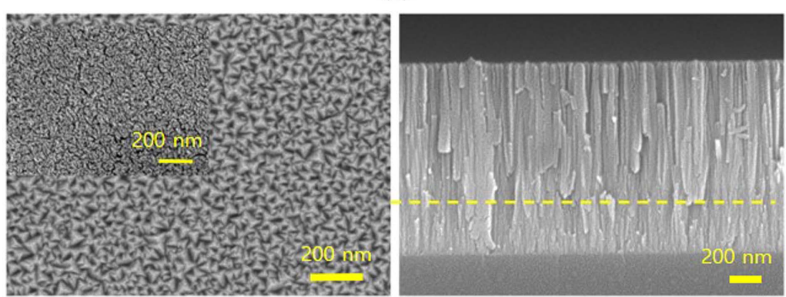

(b)
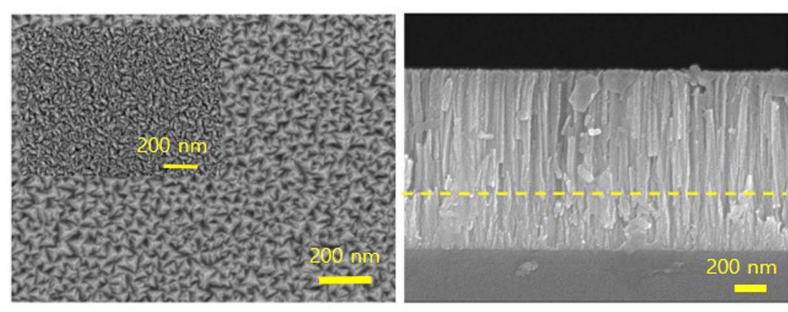

(c)
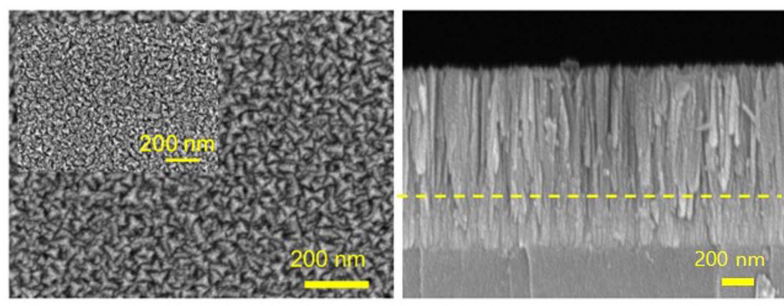

(d)

Figure 4. Plane-view and cross-sectional FE-SEM images of the Mo bi-layers deposited at various substrate temperatures: (a) 200, (b) 300, (c) 400 and (d) $500^{\circ} \mathrm{C}$.

can be expressed as [11]

where $\rho$ is the resistivity of film, $\rho_{o}$ that of an infinitely thick film, $\lambda$ is the ratio of the film thickness $t$ to the mean free path $l$ of the bulk and $p$ is the specularity parameter. The measured $\rho$ can be compared with theoretical results due to FS theory; using well-known published data (e.g. ref [14], this value was calculated for a mean free path $l_{293 \mathrm{~K}}$ of $39.5 \mathrm{~nm}[12,13]$ and a coefficient $p$ from the surface of zero. Our experimental data can be interpreted using FS theory, the resistivity of the films decreased as the thickness increased from $1.06 \mu \mathrm{m}$ to $1.39 \mu \mathrm{m}$.

The electrical properties of the Mo films improved as the strain decreased. The effect of the various substrate temperatures on the electrical resistivity was studied in an attempt to find the deposition conditions that could be used to obtain low resistivity Mo thin films. Figure 6 shows the inverse relationships of the strain and the resistivity to the grain size of the Mo films with different growth temperatures. We found that, the resistivity of the Mo films

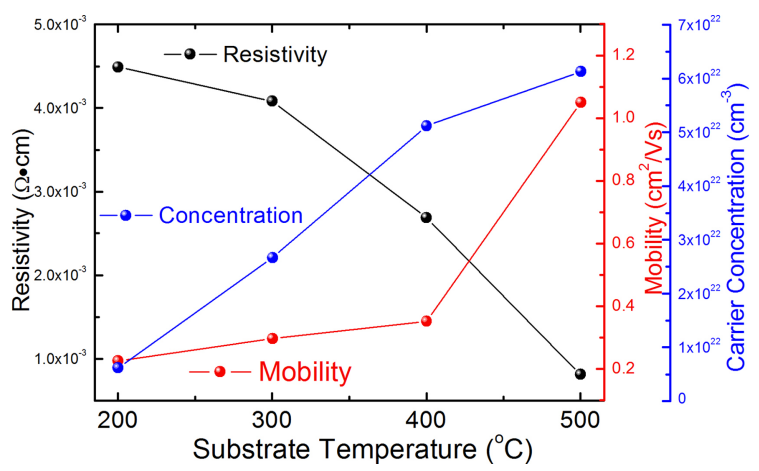

Figure 5. Electrical resistivity, carrier mobility, and carrier concentration dependencies on the substrate temperature, which are from Hall measurements at room temperature. (a) single layer (b) bi-layer.

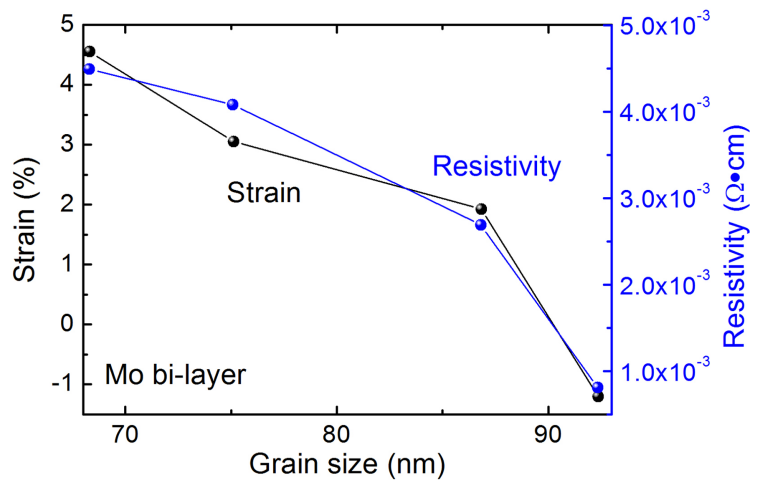

Figure 6. The inverse relationship of the strain and resistivity to the grain size of the Mo films with different growth temperatures.

decreases with increasing substrate temperature, which is accompanied by an increase of the grain size. From the above results of Hall measurements of the increase of the grain size with increasing the carrier concentration of the Mo film, we are able to explain this behavior. The increase of the grain size of the Mo films induces a reduction of the grain boundary potential barrier height, as well as of the number of grain boundaries the carrier has to cross during electrical transport, which in turn leads to a decrease of the resistivity. And, because the strain is a manifestation of a dislocation network in the films, a decrease of strain indicates a decrease of the concentration of the lattice imperfections and the formation of high quality films with increasing grain size and improvement in the crystallinity, with fewer defects [15].

\section{Conclusions}

Molybdenum thin films were grown on soda-lime glass using a DC magnetron sputtering system. The effects of the deposition parameters, such as the substrate temperature, on the lattice constant, grain size, strain and resistivity of the Mo thin films have been investigated. The main goal of this work was to clearly identify the deposition conditions, something lacking in the literature, that could ensure the 
deposition of Mo bi-layers with good adhesion to the SLG, lower resistivity and good reproducibility. The XRD results show that the crystallites of the films are mainly oriented in the (110) direction. Hall measurements using the van der Pauw configuration indicated that it is possible to obtain low resistivity Mo films by appropriately increasing the substrate temperature. From the FE-SEM images, we found that the resistivity of the Mo films decreases with increasing substrate temperature, which is accompanied by an increase of the grain size. In conclusion, we have deposited an Mo bi-layer film having a better crystallinity and a lower resistivity; this film can be suitably used as a back-contact layer in CIGS solar cells.

\section{Acknowledgements}

This work was supported by the Basic Science Research Program (NRF-2011-0024709) through the National Research Foundation of Korea (NRF), funded by the Ministry of Education, Science and Technology and the Priority Research Centers Program (2009-0093818).

\section{Refereneces}

[1] A. Rockett and R. W. Barkmire J. Appl. Phys. 70 R81 (1991).

[2] P. Jackson, D. Hariskos, E. Lotter, S. Paetel, R. Wuerz, R. Menner, W. Wischmann, and M. Powalla Prog. Photovolt.: Res. Appl. 19 894 (2011).

[3] T. J. Vink, M. A. J. Somers, J. L. C. Daams, and A. G. Dirks J. Appl. Phys. 704301 (1991).

[4] H. Khatri and S. Marsillac J. Phys.: Comdens. Matter 201 (2008)

[5] T. P. Drüsedau, F. Klabunde, P. Veit, and T. Hempel Phys. Status Solidi(a) 161167 (1997).

[6] R. F. Bunshah 1994 Handbook of Deposition Technologies for Films and Coatings-Science, Technology and Applications $2^{\text {nd }}$ edn, (New York: William Andrew Publishing/Noyes).

[7] D. M Mattox1998 Handbook of Physical Vapor Deposition (PVD) Processing, (New York: William Andrew Publishing/Noyes).

[8] M. A, Green 2006 Prog. Photovolt: Res. Appl. 14383.

[9] Rudmann Dominik 2004 "Effects of sodium on growth and properties of $\mathrm{Cu}(\mathrm{In}, \mathrm{Ga}) \mathrm{Se}_{2}$ thin films and solar cells" DISS. ET, Nr. 15576.

[10] S. A. Pethe, E. Takahashi, A. Kaul, and N. G. Dhere 2012 Solar Cells 1001

[11] D. Larson 1971 "Physics of Thin Films" Vol.6, edited by M. Francomb and R. W. Hoffman (Academic Press, New York).

[12] E. Fawcett and D. Griffiths 1962 J. Phys. Chem. Solids 231631.

[13] D. P. Almond, D. A. Detwiler, and J. A. Payne 1975 Phys. Lett. A 54229.

[14] T. J. Coutts and J. E. Morris 1974 Thin Solid Films 471.

[15] M. Öztas 2008 Chin.Ohys.Lett 2511. 